\title{
Utilisation des données enzymatiques et mor- phologiques pour l'étude des populations et de la domestication des plantes : I. Séparation et iden- tification génétique d'isozymes chez le mil (Pen- nisetum typhoides Bur. Stapf \& Hubb.)
}

\author{
Nejib TRIGUI, Michel SANDMEIER, Marcel SALANOUBAT \& Jean PERNES \\ C.N.R.S., Génétique et Physiologie du Développement des Plantes, F 91190 Gif-sur-Yvette
}

RÉSUMÉ

\begin{abstract}
Des données acquises au cours du temps pour de nombreux marqueurs morphologiques et enzymatiques chez le mil (Pennisetum typhoïdes) permettent d'entreprendre des études sur la structure génétique des populations et la domestication de cette céréale. Dans ce premier travail, les conditions techniques optimales pour l'analyse de 12 systèmes enzymatiques (*ADH, MDH, ICD, 6-PGD, CAT, POX, GOT, AK, PGM, EST, PHOS et PGI) chez le mil sont décrites. Des déterminismes génétiques sont proposés pour certains systèmes à partir des zymogrammes des parents et des hybrides $F_{1}$; pour les autres, ces déterminismes ont été vérifiès sur des ségrégations en $\mathrm{F}_{2}$. Le nombre d'allèles présents à chaque locus a été recensé chez de nombreuses populations provenant d'Afrique.
\end{abstract}

Mots clés additionnels : Electrophorèse, isozymes, déterminisme génétique, locus, allèle.

Utilization of enzymatic and morphological data for the study of populations and the domestication of plants: 1. Separation and genetic identification of isozvmes of pearl millet (Pennisetum typhoides).

Morphological and enzymatic data on pearl millet (Pennisetum typhoides) have allowed sudies on the population genetic structure and domestication of this cereal. In this first report, the technicai conditions for the analysis of 12 enzyme systems (ADH, MDH, ICD, 6-PGD, CAT, POX, GOT, AK, PGM, EST, PHOS and PGI) in pearl millet have been described. The inheritance of some of the systems has been proposed from zymograms of parents and hybrids $F_{1}$, while the inheritance of others has been established. The number of alleles present at each locus has been recorded in many African populations of pearl millet.

Additional key words : Electrophoresis, isozymes, inheritance, locus, allele.

* ADH : Alcool déshydrogénase

MDH : Malate déshydrogénase

ICD : Iso-citrate déshydrogénase

6-PGD : Phosphogluconate déshydrogénase

CAT : Catalase

POX : Péroxydase

$\begin{array}{ll}\text { GOT } & \text { Glutamate oxalo-acétate transaminase } \\ \text { AK } & \text { : Adénylate Kinase } \\ \text { PGM } & \text { Phosphoglucomutase } \\ \text { EST } & \text { Estérase } \\ \text { PHOS } & \text { Phosphatase } \\ \text { PGI } & \text { Phosphoglucoisomérase }\end{array}$

en évidence leur structure et leur physiologie. Ils peuvent également être utilisés pour l'étude de processus évolutifs (exemple : la domestication, PERNÈs, 1983) ou les perturbations provoquées par l'androgenèse

(Bui Dang Ha \& Pernès, 1982).
L'étude de la diversité génétique des populations de mil nécessite l'identification de nombreux marqueurs morphologiques et enzymatiques permettant de mettre 
Cette recherche de marqueurs enzymatiques a été entreprise chez le mil (Pennisetum typhoïdes Bur. Stapf \& Hubb.) depuis quelques années déjà (LEBLANC, 1978 ; SANDMEIER et al., 1981 ; LEBLANC \& PERnÈ, 1983 ; TRIGUi, 1984 ; TOSTAIN \& RIANDEY, 1984 et 1985). Ces travaux ont porté sur ADH, estérases, peroxydases, $\mathrm{MDH}$, catalase et endopeptidases.

Nous avons augmenté le nombre d'enzymes étudiées (12 au total), en adaptant au mil les méthodes classiques utilisées par d'autres auteurs (ORNSTEIN, 1964 ; STUBER et al., 1977 ; CARDY et al., 1980). Les zymogrammes obtenus ont permis de déterminer la structure quaternaire des formes actives des enzymes (monomère, dimère...) et les interactions entre locus ou formes alléliques différentes. Les allèles ont été dénombrés, pour chaque locus, en étudiant de nombreuses populations de mil.

\section{MATÉRIEL ET MÉTHODES}

\section{A. Préparation des extraits}

Les protéines sont extraites soit à partir de feuilles bien développées (plantes âgées de 2 à 10 semaines) (SANDMEIER et al., 1981), soit à partir de jeunes plantules étiolées de $3 \mathrm{j}$, mises en culture à $25-27^{\circ} \mathrm{C}$, à l'obscurit: (TRIGUI, 1984).
Les feuilles sont broyées dans un tampon tris- $\mathrm{HCl}$ $(0,2 \mathrm{M} \mathrm{pH} \mathrm{8,5),} \mathrm{contenant} 0,03 \mathrm{p}$. 100 de 2-mercaptoéthanol $(\mathrm{V} / \mathrm{V})$ et $0,58 \mathrm{M}$ de saccharose. On utilise $1 \mathrm{ml}$ de tampon pour $0,4 \mathrm{~g}$ de feuilles. Pour les jeunes plantules, on prélève les coléoptiles qui sont broyés individuellement dans $50 \mu \mathrm{l}$ d'ascorbate de $\mathrm{Na}$ $(0,42 \mathrm{M}, \mathrm{pH} 7,4)$ contenant $0,5 \mathrm{M}$ de saccharose. Cette technique nous permet de récupérer les plantules et d'analyser certains isoenzymes sur les feuilles.

Dans les 2 cas, les extraits sont centrifugés à $4{ }^{\circ} \mathrm{C}$ à $30000 \mathrm{~g}$ pendant $15 \mathrm{mn}$. Ils peuvent être utilisés frais ou conservés pendant quelques jours au froid $\left(-18^{\circ} \mathrm{C}\right)$.

\section{B. Méthodes d'électrophorèse}

\section{Amidon}

Les méthodes utilisant l'amidon comme support sont décrites dans les travaux de SCANDAlios (1969), Stuber et al., (1977), CARDY et al. (1980). La composition des tampons et les conditions de migration sont données dans le tableau 1.

Les gels sont coulés dans des moules de plexiglas dont les dimensions sont les suivantes :

$\begin{array}{llll}\text { - Histidine } & : 17,5 & \text { (front de migration) } & \times 19,5 \times 1 \mathrm{~cm} \\ \text { - Lithium-Borate }: 17,5 & \text { (front de migration) } & \times 14,5 \times 1 \mathrm{~cm} \\ \text { - Tris-Maléique } & : 11,5 & \text { (front de migration) } & \times 22,0 \times 1 \mathrm{~cm}\end{array}$

Dans les 2 premiers cas, on introduit des rectangles de papier Whatman $(3 \mathrm{MM}, 3 \times 12 \mathrm{~mm})$ imbibés

TABLEAU 1

Composition des tampons d'électrophorèse et conditions de migration.

Composition of electrophoresis buffers and migration conditions.

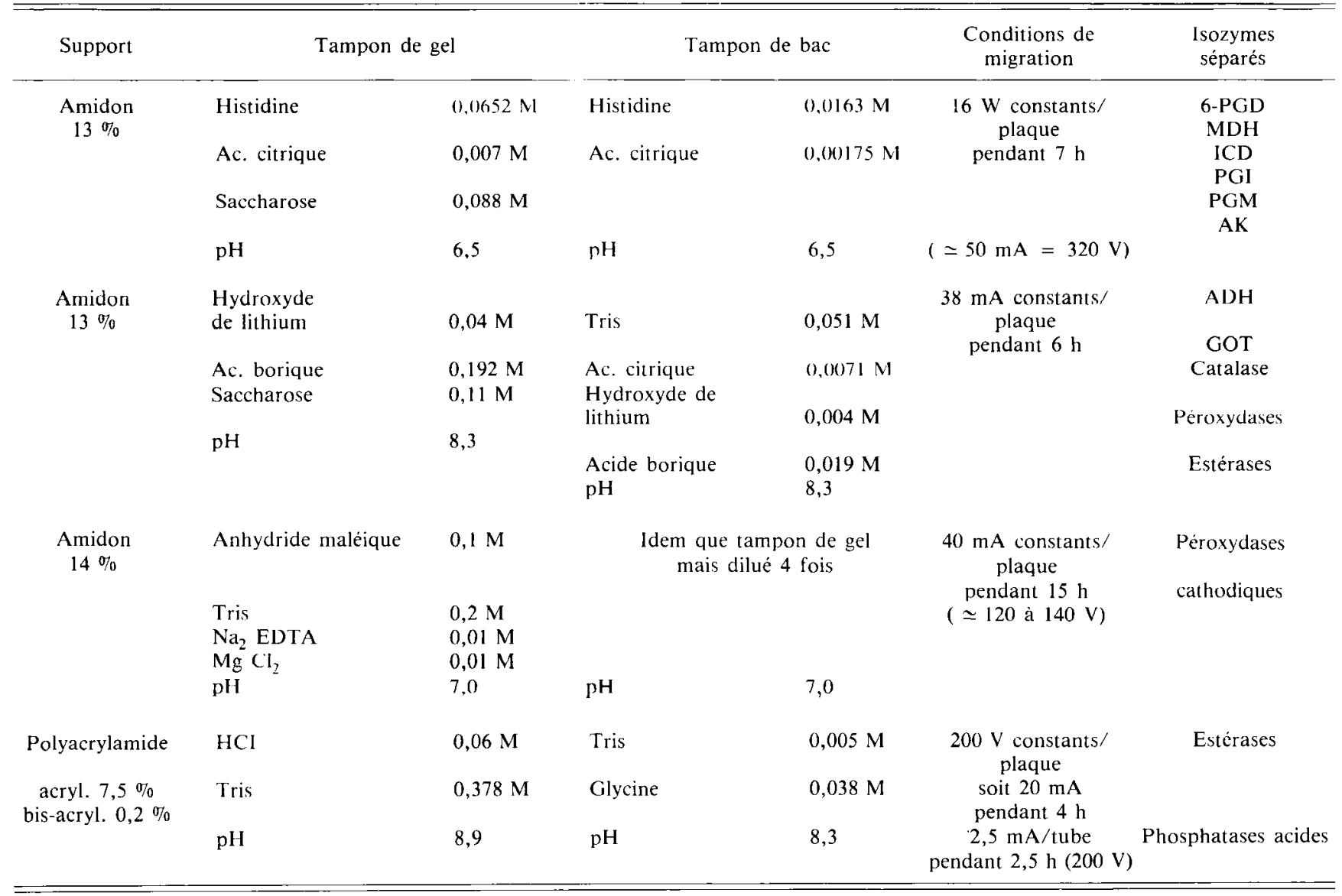


d'extrait $(10 \mu$ l environ) dans des fentes préalablement découpées dans l'épaisseur du gel (24 extraits/plaque). Pour le $3^{\mathrm{e}}$ système (tris-Maléique), on place un peigne dans l'épaisseur du gel au moment de sa préparation ; on le retire quand le gel est froid et on a ainsi 10 alvéoles $/$ gel $(2 \times 7 \times 10 \mathrm{~mm})$ permettant de recevoir $50 \mu \mathrm{l}$ d'extrait chacune.

Les électrophorèses sont réalisées en chambre froide $\left(5^{\circ} \mathrm{C}\right)$ et on place de la glace sur le gel pour éviter tout échauffement.

\section{Polyacrylamide}

On utilise la méthode d'OrNSTEIN (1964). Les catalyseurs de polymérisation utilisés sont le persulfate d'ammonium $(0,003 \mathrm{M})$ et le Temed $(0,6 \mathrm{ml} / \mathrm{l})$. La séparation des enzymes se fait sur plaque verticale (Matériel BIO-RAD), dans laquelle on a aménagé 20 alvéoles qui peuvent contenir $50 \mu \mathrm{l}$ d'extrait chacune.

Les phosphatases acides sont séparées sur tubes d'acrylamide, qui sont préparés de la même façon que les plaques (tabl. 1) (7,5 p. 100 acrylamide $+0,2$ p. 100 de bis-acrylamide) ; chaque boudin reçoit $100 \mu \mathrm{l}$ d'extrait. Les migrations sont réalisées en chambre froide $\left(5^{\circ} \mathrm{C}\right)$.

\section{Révélation des isoenzymes}

La séparation électrophorétique étant terminée, on procède à la révélation des diverses enzymes. En ce qui concerne l'amidon, on coupe des tranches de $1,5 \mathrm{~mm}$ d'épaisseur au moyen d'un fil d'acier de $6 / 100$ de $\mathrm{mm}$. Chaque tranche nous permet de révéler une enzyme. Ainsi nous arrivons à révéler jusqu'à 6 enzymes par électrophorèse. La révélation se fait en plaçant la tranche de gel dans une boîte de Petri de $20 \mathrm{~cm}$ de diamètre avec $50 \mathrm{ml}$ de solution de révélation (voir tabl. 2).

Pour l'acrylamide, les «boudins » ou les plaques sont démoulés et placés directement dans le milieu de révélation (tabl. 2).

\section{Codage des isoenzymes}

En tenant compte des recommandations de la Commission internationale de Nomenclature (1973), «IUPAC-IUB »: Commission on Biochemical Nomenclature $(\mathrm{CBN})$, nous avons codé les isoenzymes du mil de la façon suivante :

1. La dénomination des locus d'un système enzymatique donné, désignés par des lettres, se fait suivant la mobilité des bandes vers l'anode, la plus lente étant désignée par la lettre $\mathrm{A}$. En cas de migration cathodique, on utilise le même principe de codage, mais la lettre est précédée du signe $(-)$ moins. - A est la bande qui migre le moins loin vers la cathode.

2. Pour un locus donné, les allèles sont classés suivant leur mobilité électrophorétique, $(1,2,3$, etc...). L'indice le plus élevé désignera l'allèle qui migre le plus loin vers l'anode. L'indice 0 (zéro) est réservé à un éventuel allèle nul.

\section{E. Matériel biologique}

Les populations, cultivars ou variétés de mil analysés ont pour origine :

- L'Afrique de l'Ouest (20 populations) : il s'agit de semences prospectées par l'ORSTOM (CLÉMENT, 1985), au Mali, Sénégal, Niger et Burkina-Faso.

- L'Afrique du Nord (30 populations) : il s'agit essentiellement de cultivars prospectés par TRIGUI (1984) en Tunisie (25 populations), les autres venant d'Algérie ou du Maroc.

- L'Afrique de l'Est : les 8 populations viennent du Kenya, de Tanzanie et du Soudan.

Nous avons analysé aussi bien des mils cultivés que des formes spontanées (Afrique sub-saharienne uniquement).

\section{F. Principe des interprétations génétiques}

Le déterminisme génétique des systèmes enzymatiques que nous proposons est supposé à partir des observations de zymogrammes des parents et hybrides $F_{1}$. Cependant ce déterminisme a été vérifié, pour certains systèmes (voir tabl. 3), par l'analyse de ségrégations en $\mathrm{F}_{2}$.

\section{RÉSULTATS}

$\mathrm{ADH}, \mathrm{GOT}$ et CAT ne sont révélés que chez les jeunes plantules étiolées, alors que les POX, AK, PHOS et MDH A sont analysés sur des feuilles vertes uniquement. Les autres isozymes sont révélés aussi bien sur les feuilles que sur les plantules étiolées.

Les locus, les allèles inventoriés et éventuellement leur déterminisme génétique sont regroupés dans le tableau 2. Les ségrégations obtenues pour 10 locus sont résumées dans le tableau 3.

Les résultats obtenus avec les 12 systèmes enzymatiques étudiés sont les suivants :

\section{A. Les oxydoréductases}

1. Alcool déshydrogénase, ADH (Alcohol : NAD oxidoréductase, EC 1.1.1.1.) (fig. 1)

Cette enzyme a été étudiée chez le mil (LEBLANC, 1978 ; BanUETT-BOURILLON \& HAGUE, 1979 ; LEBLANC \& PERNĖ, 1983 ; LEFRANC-RIANDEY, 1984). Son déterminisme génétique est bien connu : en effet 2 locus (A et B) codent pour cette enzyme. En conditions aérobies, seuls les produits du locus $\mathrm{A}$ et de l'interaction entre les locus A et B sont actifs. Les interactions interlocus indiquent le type d'allèle du locus B muet en aérobie. L'ADH est un dimère et nous avons récensé 3 allèles pour chacun des 2 locus dans l'ensemble des populations étudiées. 
TABLEAU 2

Révélation des isozymes chez le mil : leur structure et leur déterminisme génétique.

Resolution of pearl millet isozymes : their structure and genetic control.

\begin{tabular}{|c|c|c|c|c|c|}
\hline Enzymes & Locus & Allèles & $\begin{array}{l}\text { Structure de la } \\
\text { protéine }\end{array}$ & $\begin{array}{l}\text { Ségrég. en } \\
\mathrm{F}_{2}\end{array}$ & Milieu de révélation \\
\hline AK & $\begin{array}{l}\text { A } \\
\mathrm{B} \\
\mathrm{C}\end{array}$ & $\begin{array}{l}0,1 \\
0,1 \\
1^{*}\end{array}$ & $?$ & $?$ & $\begin{array}{l}\text { Glucose } 15 \mathrm{mM} ; \text { NADP } 0,4 \mathrm{mM} ; \text { ADP } 1,2 \mathrm{mM} \text {; } \\
\mathrm{Mg} \mathrm{Cl}_{2} 5 \mathrm{mM} ; \mathrm{MTT} 0,5 \mathrm{mM} ; \mathrm{PMS} 0,25 \mathrm{mM} ; \\
\text { Hexokinase } 450 \text { unités } / \mathrm{L} ; \mathrm{G}-6-\mathrm{PD} 2000 \text { unités } / \mathrm{l} \\
\text { Tampon tris- } \mathrm{HCl}, \mathrm{pH} 8,5,0,1 \mathrm{M} \\
37^{\circ} \text { obscurité }\end{array}$ \\
\hline $\mathrm{ADH}$ & $\begin{array}{l}\text { A } \\
\text { B }\end{array}$ & $\begin{array}{l}1,2^{*}, 3 \\
1,2^{*}, 3\end{array}$ & dimère & $\begin{array}{l}(1-2-1) \\
(1-2-1)\end{array}$ & $\begin{array}{l}\text { Ethanol } 170 \mathrm{mM} \text {; NAD } 0,35 \mathrm{mM} ; \mathrm{Mg} \mathrm{Cl}_{2} 5 \mathrm{mM} \text {; } \\
\text { MTT } 0,5 \mathrm{mM} ; \mathrm{PMS} 0,065 \mathrm{mM} ; \\
\text { Tampon tris-HCl, pH } 8,5,0,1 \mathrm{M} \\
37^{\circ} \text { obscurité }\end{array}$ \\
\hline CAT & A & $1^{*}, 2^{*}$ & tétramère & $(1-2-1)$ & $\begin{array}{l}\text { a) } 10 \text { min dans } \mathrm{O}_{2} \mathrm{H}_{2} 110 \text { vol. } 450 \mu \mathrm{l} / 1 \\
\text { b) hexocyanoferrate de K. } 30 \mathrm{mM} ; \mathrm{FeCl}_{3} 37 \mathrm{mM} \text {; } \\
20^{\circ} \text { obscurité }\end{array}$ \\
\hline EST- & $\begin{array}{l}\text { A } \\
\text { B } \\
\text { C } \\
\text { D }\end{array}$ & $\begin{array}{l}0^{*}, 1 \\
0,1^{*} \\
0,1^{*} \\
0^{*}, 1\end{array}$ & $?$ & $?$ & $\begin{array}{l}\text { 1-naphtyl-acétate } 5,4 \mathrm{mM} \text { (locus A-B-D) } \\
\text { ou 2-naphtyl-acétate } 5 \mathrm{mM} \\
\text { (locus A-C); Fast blue RR } 3,3 \mathrm{mM}: \mathrm{CaCl}_{7} 7 \mathrm{mM} \text {; } \\
\text { Tampon phosphate de } \mathrm{Na} \text { pH } 6,50,1 \mathrm{M} \\
37^{\circ} \text { obscurité }\end{array}$ \\
\hline EST & $\mathrm{E}$ & $\begin{array}{l}1,2,3,4 \\
5,6,7\end{array}$ & dimère & $(1-2-1)$ & $\begin{array}{l}\text { Acétate d'indoxyle } 1,15 \mathrm{mM} \text {; acétate cuivrique } \\
0,1 \mathrm{mM} ; \text { tampon Véronal, } \mathrm{pH} 8,2,0,05 \mathrm{M} \text {; } \\
20^{\circ} \text { obscurité }\end{array}$ \\
\hline GOT & $\begin{array}{l}\text { A } \\
\text { B } \\
\text { C }\end{array}$ & $\begin{array}{l}1,2^{*}, 3 \\
1,2^{*} \\
1^{*}\end{array}$ & dimère & & $\begin{array}{l}\text { Ac. aspartique } 30 \mathrm{mM} \text {; ac. } \alpha \text {-cétoglutarique } 14 \mathrm{mM} \text {; } \\
5 \text {-P-Pyridoxal } 0,1 ; \mathrm{mM} \text {, Fast blue BB } 13,3 \mathrm{mM} \text {; } \\
\text { Tampon tris- } \mathrm{HCl}, \mathrm{pH} 8,5,0,25 \mathrm{M} \\
20^{\circ} \text { obscurité }\end{array}$ \\
\hline ICD & A & $1,2^{*}, 3$ & dimère & $?$ & $\begin{array}{l}\text { Isocitrate de } \mathrm{Na} 12 \mathrm{mM} ; \mathrm{NADP} 0,4 \mathrm{mM} ; \\
\mathrm{Mg} \mathrm{Cl}_{2} 5 \mathrm{mM} ; \mathrm{NBT} 0,25 \mathrm{mM} ; \mathrm{PMS} 0,07 \mathrm{mM} ; \\
\mathrm{PVP} 40 \mathrm{~T} 6 \mathrm{~g} / 1 ; \\
\text { Tampon tris-HCl, pH } 8,5,0,1 \mathrm{M} \\
37^{\circ} \text { obscurité }\end{array}$ \\
\hline $\mathrm{MDH}$ & $\begin{array}{r}-A \\
\text { A } \\
\text { B } \\
\text { C } \\
\text { D }\end{array}$ & $\begin{array}{l}1,2 \\
1,2^{*}, 3 \\
1,2,3^{*}, 4 \\
5 \\
1,2^{*} \\
1,2^{*}, 3\end{array}$ & dimère & $(1-2-1)$ & $\begin{array}{l}\text { Malate de } \mathrm{Na} 100 \mathrm{mM} ; \mathrm{NAD} 0,35 \mathrm{mM} ; \mathrm{MgCl}_{2} 5 \mathrm{mM} \\
\text { MT } 0,5 \mathrm{mM} ; \mathrm{PMS} 0,07 \mathrm{mM} ; \\
\text { Tampon tris-HCl, pH 8,5,0,1 M } \\
37^{\circ} \text { obscurité }\end{array}$ \\
\hline $\begin{array}{l}\text { POX- } \\
\text { cathod. }\end{array}$ & $\begin{array}{l}-\mathrm{J} \\
-\mathrm{I} \\
-\mathrm{H} \\
\\
-\mathrm{G} \\
-\mathrm{F} \\
-\mathrm{E} \\
-\mathrm{D} \\
-\mathrm{C} \\
-\mathrm{B} \\
-\mathrm{A}\end{array}$ & $\begin{array}{l}0,1^{*} \\
0,1^{*} \\
0^{*}, 1 \\
\\
0,1 \\
1^{*} \\
0^{*}, 1 \\
0,1 \\
0,1 \\
0,1 \\
0,1\end{array}$ & monomère & $\begin{array}{l}- \\
- \\
(3-1) \\
- \\
- \\
-\end{array}$ & $\begin{array}{l}\text { Gaicol } 90 \mathrm{mM} \text {; } \\
\text { 3-amino-9-éthylcarbazole } 2,4 \mathrm{mM} \text {; } \\
\text { diméthylformamide } 50 \mathrm{ml} / \mathrm{l} \text { (pour dissoudre le } 3 \text {-amino-9- } \\
\text { éthylcarbazole) } \\
\mathrm{O}_{2} \mathrm{H}_{2} 30 \text { volumes } 5 \mathrm{ml} / 1 \text {; } \\
\mathrm{CaCl}_{2} 2 \mathrm{mM} \text {; } \\
\text { Tampon acétate de } \mathrm{Na}, \mathrm{pH} 5,0,0,05 \mathrm{M} \\
20^{\circ} \text { obscurité }\end{array}$ \\
\hline $\begin{array}{l}\text { POX- } \\
\text { anod. }\end{array}$ & $\begin{array}{l}\text { A } \\
\text { B } \\
\text { C } \\
\text { D } \\
\text { E } \\
\text { F } \\
\text { G } \\
\text { H }\end{array}$ & $\begin{array}{l}0^{*}, 1 \\
0,1^{*} \\
0^{*}, 1 \\
0^{*}, 1 \\
0^{*}, 1 \\
1^{*} \\
0^{*}, 1 \\
0,1^{*}\end{array}$ & monomère & $\begin{array}{c}(3-1) \\
(3-1) \\
(3-1 \text { ou } 7-9)\end{array}$ & $\begin{array}{l}\text { 1) Incubation pendant } 20 \mathrm{~min} \text {. dans gaïcol } 90 \mathrm{mM} \text {; } \\
\text { Tampon acétate de } \mathrm{Na} 0,1 \mathrm{M}, \mathrm{pH} 4,0 \\
\text { Jeter le milieu et placer dans } \\
\text { 2) 3-amino-9-éthylcarbazole } 2,4 \mathrm{mM} \text { dissous par } \\
\text { diméthylformamide } 50 \mathrm{ml} / 1 ; \mathrm{O}_{2} \mathrm{H}_{2} 30 \text { volumes } \\
5 \mathrm{ml} / \mathrm{l} ; \mathrm{CaCl} 2 \mathrm{mM} ; \\
\text { Tampon acétate de } \mathrm{Na}, \mathrm{pH} 5,00,05 \mathrm{M} \\
20^{\circ} \text { obscurité }\end{array}$ \\
\hline PHOS & $\begin{array}{l}\text { A } \\
B \\
C \\
D \\
E \\
F\end{array}$ & $\begin{array}{l}0^{*}, 1 \\
1^{*} \\
1^{*} \\
0^{*}, 1 \\
0^{*}, 1 \\
0^{*}, 1\end{array}$ & $?$ & $(3-1)$ & $\begin{array}{l}\text { 1-naphtyl-phosphate, } 4 \mathrm{mM} \\
\text { 2-naphtyl-phosphate, } 4 \mathrm{mM} \\
\text { Fast blue BB } 3,3 \mathrm{mM} ; \\
\text { Tampon acétate de } \mathrm{Na}, 0,1 \mathrm{M}, \mathrm{pH} 4,0 \\
37^{\circ} \text { obscurité }\end{array}$ \\
\hline 6-PGD & $\begin{array}{l}\text { A } \\
\text { B } \\
\text { C }\end{array}$ & $\begin{array}{l}1,2^{*}, 3 \\
0,1^{*} \\
1^{*}\end{array}$ & $\begin{array}{l}\text { dimère } \\
\text { dimère }\end{array}$ & $(1-2-1)$ & $\begin{array}{l}\text { Ac. 6-phosphogluconique } 0,7 \mathrm{mM} \\
\mathrm{MgCl}_{2} 5 \mathrm{mM} ; \mathrm{NADP} 0,15 \mathrm{mM} ; \\
\mathrm{MTT} 0,5 \mathrm{mM} ; \text { PMS } 0,07 \mathrm{mM} ; \\
\text { Tampon tris- } \mathrm{HCl} 0,1 \mathrm{M} . \mathrm{pH} 8,5 \\
37^{\circ} \text { obscurite }\end{array}$ \\
\hline PGI & A & $\begin{array}{l}1,2^{*}, 3 \\
4^{*}, 5\end{array}$ & dimère & $(1-2-1)$ & $\begin{array}{l}\text { Fructose-6-P } 2,5 \mathrm{mM} \text {; glucose 1-P, } 16 \mathrm{mM} \text {; } \\
\text { NADP } 0,15 \mathrm{mM} ; \mathrm{MgCl}_{2} 5 \mathrm{mM} ; \\
\text { MTT } 0,5 \mathrm{mM} ; \text { PMS } 0,07 \mathrm{mM} ;\end{array}$ \\
\hline PGM & $\Lambda$ & $\begin{array}{l}1,2^{*} \\
3,4^{*}\end{array}$ & monomère & $(1-2-1)$ & $\begin{array}{l}\mathrm{Na}_{2} \text { EDTA } 2,7 \mathrm{mM} \text {; } \\
\text { Gilucose-6-P-déshydrogénase } 2000 \text { unites/1; } \\
\text { Tampon tris-HCl } 0,1 \mathrm{M}, \mathrm{pH} 8,5 \\
37^{\circ} \text { obscurité }\end{array}$ \\
\hline
\end{tabular}


TABLEAU 3

Ségrégation en $F_{2}$ de 10 locus.

$F_{2}$ segregations for 10 loci.

\begin{tabular}{lcccc}
\hline \hline \multicolumn{1}{r}{ Enzymes } & Locus & \multicolumn{2}{c}{ Ségrégation } & $\mathrm{Chi}_{2}$ \\
\hline EST & $\mathrm{E}$ & $46-83-49$ & $1 / 2 / 1$ & 0,91 \\
MDH & $\mathrm{B}$ & $78-133-53$ & $1 / 2 / 1$ & 4,75 \\
POX cat. & $-\mathrm{I}$ & $40-70$ & $7 / 9$ & 2,49 \\
POX cat. & $-\mathrm{H}$ & $90-20$ & $3 / 1$ & 2,73 \\
POX cat. & $-\mathrm{E}$ & $136-42$ & $3 / 1$ & 0,19 \\
POX anod. & $\mathrm{A}$ & $81-29$ & $3 / 1$ & 0,02 \\
PHOS & $\mathrm{A}$ & $135-30$ & $3 / 1$ & 4,09 \\
6-PGD & $\mathrm{A}$ & $68-116-56$ & $1 / 2 / 1$ & 1,47 \\
PGl & $\mathrm{A}$ & $49-79-40$ & $1 / 2 / 1$ & 1,57 \\
PGM & $\mathrm{A}$ & $47-81-40$ & $1 / 2 / 1$ & 0,81 \\
Chi 2 seuil de 1 p. 100: 1 DL : 6,63 & & \\
\multicolumn{5}{c}{2 DL $: 9,21$} \\
\hline \hline
\end{tabular}

2. Malate déshydrogénase, MDH (L-Malate : NAD oxidoréductase, EC 1.1.1.37.) (fig. 2, 3).

La figure 3 montre la complexité des zymogrammes des MDH. On compte, en effet, un locus cathodique $(-\mathrm{A})$ et 4 locus anodiques. Dans tous les cas, nous avons plusieurs allèles pour chaque locus en plus des interactions inter et intra-locus (interactions interlocus visibles uniquement entre les produits des locus présents dans le même compartiment cellulaire).

Ainsi nous constatons une interaction entre les produits du locus B ( 5 allèles) et ceux du locus C ( 2 allèles). Si les 2 locus sont à l'état homozygote, nous observons un zymogramme de 3 bandes (la structure quaternaire de la forme active de l'enzyme est sans doute dimérique). Dans le cas où l'un des 2 locus est hétérozygote, nous observons 6 bandes et dans le cas où les 2 locus sont hétérozygotes, nous observons 10 bandes. Sur certains zymogrammes le nombre de bandes est parfois réduit par chevauchement (fig. 2).

3. Isocitrate déshydrogénase, ICD (threo- $\mathrm{D}_{\mathrm{s}}$-isocitrate : NADP oxidoréductase, EC 1.1.1.42.) (fig. 4)

Cette enzyme est détectée chez les plantules étiolées et les feuilles mais dans ces dernières sa révélation est médiocre. Cette enzyme serait codée par un locus avec 3 allèles, dont $2\left(A_{1}\right.$ et $\left.A_{3}\right)$ n'ont été trouvés que dans 3 populations à la fréquence maximum de 1 p. 100 , à l'exception d'une variété nord-africaine $(f=15 \mathrm{p} .100)$. Chez les quelques structures hybrides rencontrées, l'homodimère (produit de l'allèle rare) n'est pas révélé. Deux hypothèses peuvent expliquer ce phénomène :

1. l'homodimère relatif à l'allèle rare serait inactif ;

2. la sous-unité produite par l'allèle rare aurait subi une mutation qui gênerait la formation de l'homodimère, forme active de l'enzyme.

4. Phosphogluconate déshydrogénase, 6-PGD (6phospho-D-gluconate : NADP 2.oxidoréductase, EC 1.1.1.44.) (fig. 2)

Il existe 2 niveaux de bandes (ou 2 zones) 6-PGD, aussi bien chez les feuilles que chez les plantules.

Le $1^{\text {er }}$ niveau est codé par un locus $(A)$ où nous avons recensé 3 allèles. La présence d'interaction chez les formes hétérozygotes donne à penser que cette
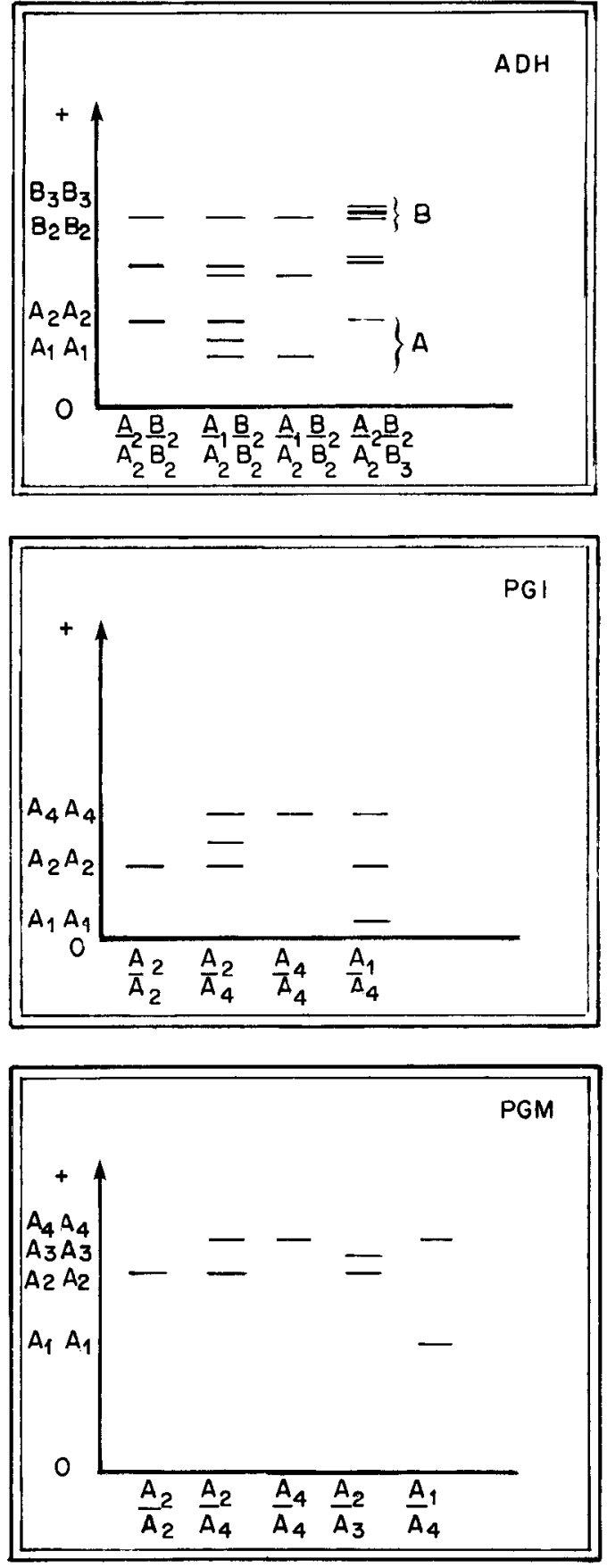

Figure 1

Zymogrammes de ADH, PGI et PGM.

Zymograms for $A D H, P G I$ and $P G M$.

enzyme est un dimère $(*)$. La ségrégation 1-2-1 obtenue en $F_{2}$ est conforme à cette situation (tabl. 3). Le $2^{\mathrm{e}}$ niveau est généralement composé de 3 bandes nécessitant l'intervention de 2 gènes. Le locus $C$ présente un seul allèle alors que le locus B en présente 2, dont l'un est nul. La bande intermédiaire est le résultat de l'interaction entre le locus $B_{1}$ et $C$. L'enzyme est un dimère. Il n'y a pas d'interaction entre les produits du locus A, d'une part, et ceux des locus B et C, d'autre part.

(*) Une bande supplémentaire a été rencontrée dans tous les zymogrammes, elle est de faible intensité et elle est peut être le résultat d'une dégradation. 

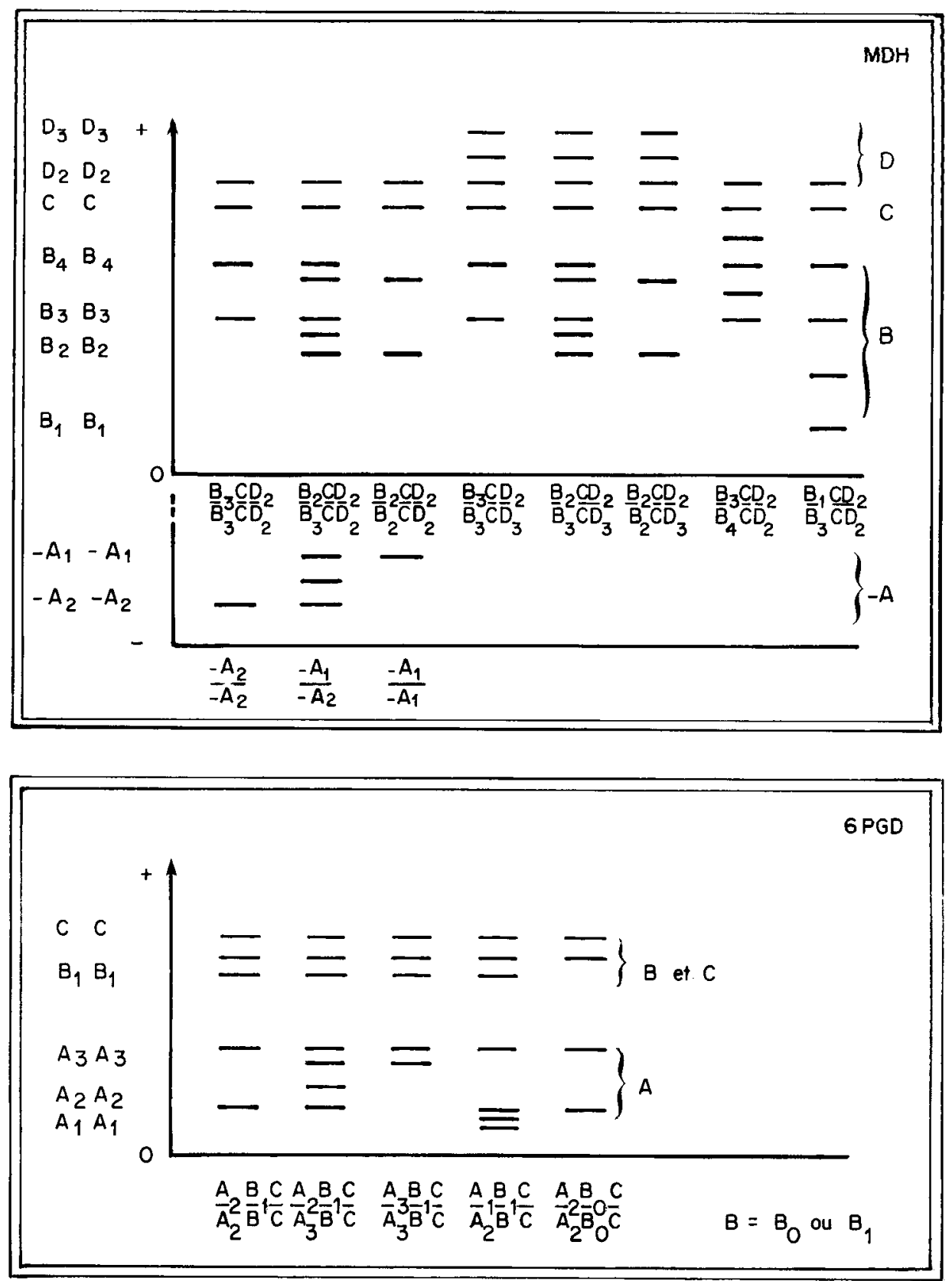

Figure 2

Zymogrammes de $M D H$ et 6-PGD.

Zymograms for $M D H$ and 6-PGD.

Exemple d'une électrophorèse de $M D H$, seuls les locus $A, B, C$ et $D$ sont révélés.

Example of $M D H$ electrophoresis, loci $A, B, C$ and $D$ only are shown. 
5. Catalase, CAT (Hydrogen-peroxide: hydrogenperoxide oxidoréductase, EC 1.11.1.6.) (fig. 4 et 5)

Dans les $1^{\text {ers }}$ stades de la germination (2 à $3 \mathrm{j}$ ), on peut mettre en évidence l'activité d'un locus Catalase avec 2 allèles (fig. 5). Les plantes hétérozygotes présentent 3 bandes intermédiaires entre les 2 bandes parentales. Nous pensons que ce sont des bandes d'interaction et que l'enzyme (la forme active) serait tétramérique comme chez le maïs (SCANDALIOS, 1979), la ségrégation en $F_{2}$ serait 1-2-1.
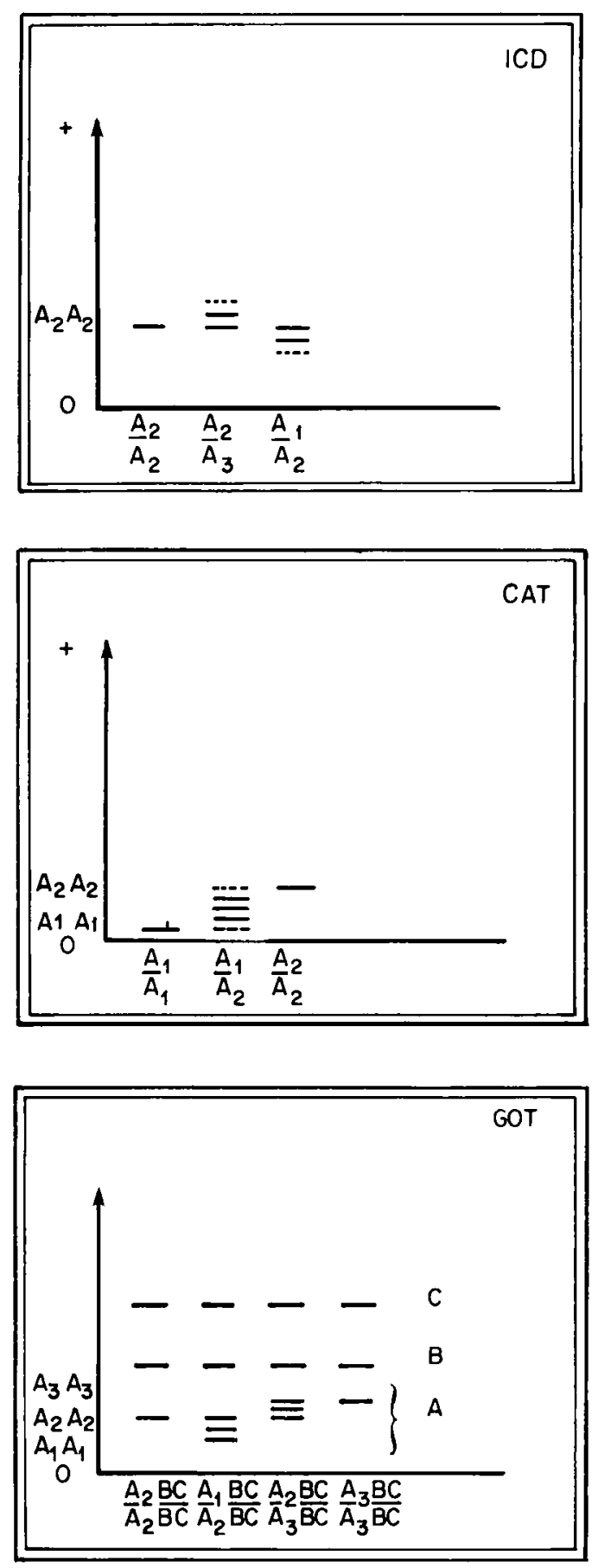

Figure 4

Zymogrammes de ICD, Catalase et GOT (pour le locus B seul l'allèle $B_{2}$ a été reporté).

Zymograms for ICD, catalase and GOT for locus B, allele $B$ only is shown).

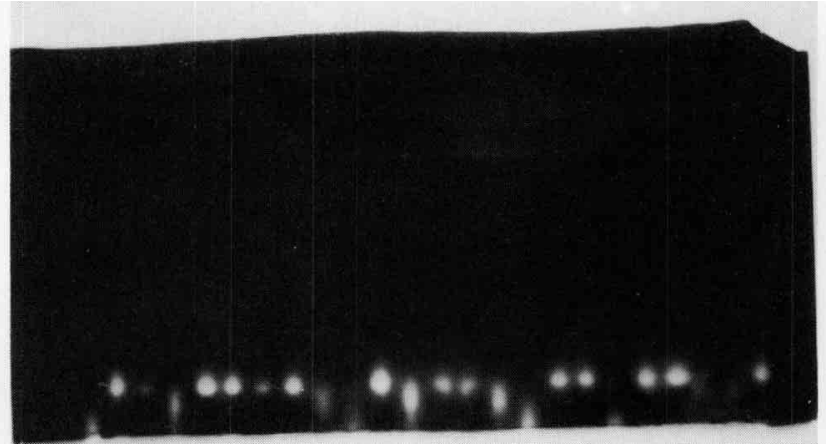

Figure 5

Exemple d'un zymogramme de Catalase où sont présentés les phénotypes $A_{1} A_{1}, A_{2} A_{2}$ et $A_{1} A_{2}$.

Example of a catalase zymogram with $A_{1} A_{1}, A_{2} A_{2}, A_{1} A_{2}$ phenotypes.

6. Péroxydases, POX (donor: $\mathrm{H}_{2} \mathrm{O}_{2}$-oxidoréductase, EC 1.11.1.7.)

Les enzymes ayant une activité péroxydasique sont très nombreuses dans les feuilles de mil, en effet nous avons pu dénombrer 18 bandes différentes.

La figure 6 montre les péroxydases cathodiques ( $-\mathrm{J}$ à $-\mathrm{A}$ ). Chaque "locus»? (bande), à l'exception de $-\mathrm{F}$, présente 2 allèles dont un est nul (absence de bandes). Pour 2 d'entre eux $(-E,-H)$, la ségrégation en $F_{2}$ est du type 3-1, elle est de 7-9 pour la bande - I (voir tabl. 2 et 3 ). La bande $-G$ varie suivant l'état physiologique de la plante, on ne peut donc pas l'utiliser comme marqueur génétique.

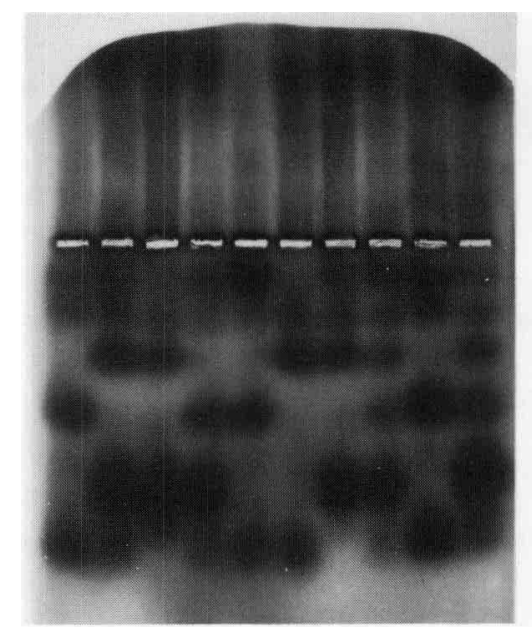

Figure 6

Exemple d'un zymogramme de péroxydases cathodiques où sont visibles les locus $-A,-C,-E,-F,-H$ et $-J$.

Example of a cathodal peroxidase zymogram where loci $-A,-C$, $E,-F,-H$ and $-J$ can be seen. 
Pour les péroxydases anodiques, seul le locus $F$ ne présente pas de polymorphisme ; la ségrégation en $F_{2}$ pour les bandes A et B est de 3-1; elle est de 3-1 (SANDMEIER et al., 1981) ou 9-7 (SALANOUBAT, 1980) pour le locus $D$ pour un croisement mettant en présence les mêmes génotypes sans que l'on ait pu déterminer les causes de ces variations (distorsion de ségrégation, réactivation d'un $2^{\mathrm{e}}$ locus...).

\section{B. Les transférases}

\section{Les aminotransférases}

Glutamate Oxaloacétate transaminase, GOT (L-Aspartate : 2-oxoglutarate aminotransférase, EC 2.6.1.1.)

Chez les jeunes germinations, 3 locus sont mis en évidence (fig. 4). Le locus A présente 3 allèles dont 2 $\left(A_{1}\right.$ et $A_{3}$ ) sont extrêmement rares (1 à 5 p. 100 chez quelques cultivars). Le locus $\mathrm{B}$ a 2 allèles, quant au locus $C$, il n'en présente qu'un seul, il est donc monomorphe. Les locus $\mathrm{A}$ et $\mathrm{B}$ présentent 3 bandes à l'état hétérozygote, cette enzyme est donc vraisemblablement un dimère. La ségrégation du locus $\mathrm{A}$ est de type 1-2-1 (tabl. 2).

\section{Les phosphotransférases}

a) Adénylate kinase, AK (ATP : AMP phosphotransférase, EC 2.7.4.3.)

Nous révélons 3 niveaux de bandes pour cette enzyme sur les feuilles vertes. Les niveaux A et B sont polymorphes avec un allèle fonctionnel et un nul (absence d'activité). Pour l'instant, nous n'avons aucune indication en ce qui concerne le déterminisme génétique de ce système enzymatique.

b) Phosphoglucomutase, PGM (-D-Glucose-1,6 diphosphate : $\alpha$-D-Glucose-1 Phosphate phosphotransférase, EC 2.7.5.1.) (fig. 1, 7)

Cette enzyme est codée par un seul locus (LEBLANC \& PERnÈs, 1983). Les structures hétérozygotes présentent 2 bandes, prouvant que la forme active de cette enzyme est monomérique. Nous avons recensé 4 allèles différents à ce locus. La ségrégation en $\mathrm{F}_{2}$ a été étudiée, elle est conforme aux proportions 1-2-1 (tabl. 3).

\section{Les hydrolases}

\section{Les estérases EST (Carboxyestérases, EC 3.1.1.1.)}

Dans les feuilles, 5 bandes EST sont mises en évidence ; 4 sont révélées sur amidon, avec l' $\alpha$ ou le $\beta$ naphtyl acétate comme substrat. Les produits du dernier locus sont séparés sur gel de polyacrylamide, avec l'acétate d'indoxyle comme substrat préférentiel.

\section{a) Estérases séparées sur gel d'amidon}

Les bandes A, B et D sont colorées en gris par l' $\alpha$ naphtyl acétate, alors que les bandes $A$ et $C$ sont colorées en rose par le $\beta$-naphtyl) acétate. L'utilisation de

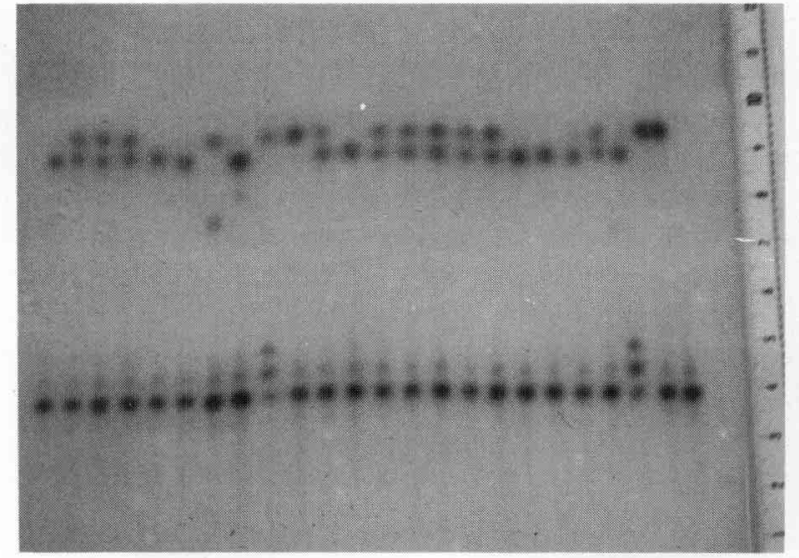

Figure 7

Exemple d'un zymogramme de PGM (en haut) et de PGI (en bas). Chez PGI, on observe les phénotypes $A_{2} A_{2}, A_{4} A_{4}, A_{2} A_{4}$ et $A_{1} A_{4}$. Chez PGM on a $A_{2} A_{2}$ et $A_{2} A_{4}$.

Example of PGM (above) and PGI (below) zymograms. For PGI, $A_{2} A_{2}, A_{4} A_{4}, A_{2} A_{4}$ and $A_{1} A_{4}$ phenotypes can be seen. For PGM, $A_{2} A_{2}$ and $A_{2} A_{4}$.

l'un ou l'autre isomère du substrat permet donc de différencier les bandes $\mathrm{B}$ et $\mathrm{C}$ qui migrent au même niveau. Le déterminisme génétique de ces bandes n'est pas encore connu.

\section{b) Estérases séparées sur gel de polyacrylamide}

La figure 8 montre 7 allèles du locus $E$ recensés chez les populations de mil étudiées. Cette série allélique présente 3 «bandes " à l'état hétérozygote correspondant à une protéine de structure dimérique. La ségrégation en $F_{2}$ est de 1-2-1 (SANDMEIER et al., 1981).

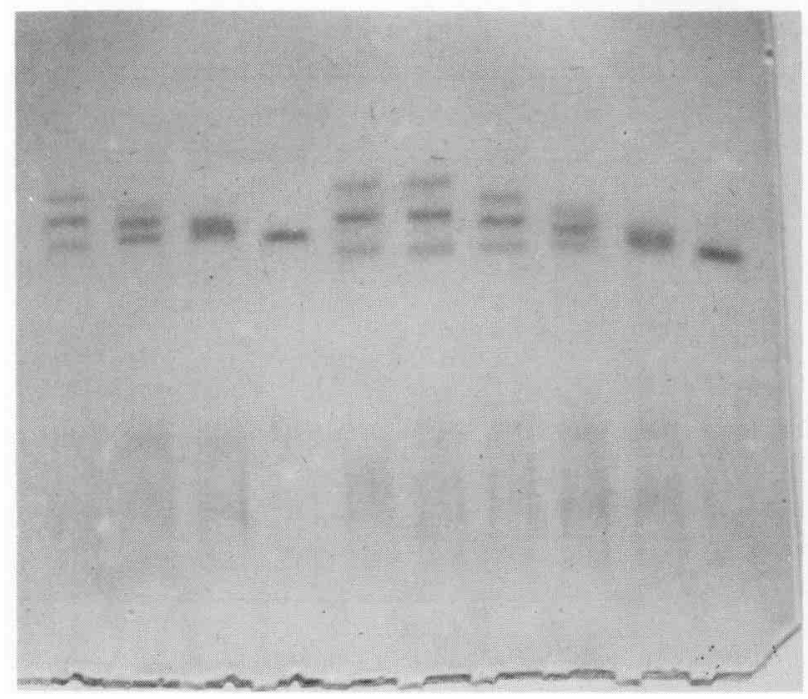

Figure 8

Estérases carboxyliques. Exemple d'un zymogramme comportant 5 allèles différents du locus $E$.

Carboxylic esterases. Example of a zymogram with 5 different alleles at locus E. 
2. Phosphatases acides, PHOS (Orthophosphoric monoester phosphohydrolase, acid optimum, EC 3.1.3.2.)

Dans les feuilles des plantes adultes, nous révélons l'activité de 4 bandes (A, D, E, F) qui ne sont pas présentes chez tous les génotypes étudiés. En plus, nous révélons 2 bandes $\mathrm{B}$ et $\mathrm{C}$ constantes quel que soit le génotype. La bande $\mathrm{A}$, semble être sous la dépendance d'un seul gène (ségrégation 3-1 en $F_{2}$ ) (tabl. 3). Nous ignorons encore le déterminisme génétique des autres bandes et la structure de cette enzyme.

\section{Les isomérases}

Phosphoglucoisomérase, PGI (D-Glucose-6Phosphate : Kétol-isomérase, EC 5.3.1.9 (fig. 1 et 7). Cette enzyme est un dimère ; elle est codée par un seul locus, la ségrégation est du type 1-2-1 en $F_{2}$ (tabl. 3).

La figure 7 montre un exemple de zymogramme de PGI (niveau bas) et les zymogrammes de PGM des mêmes individus (niveau haut). En effet, ces 2 enzymes sont révélées sur la même tranche de gel par souci d'économie.

\section{CONCLUSIONS}

Ce travail a permis de déterminer les conditions techniques optimales pour la mise en place d'une chaîne d'analyse de 12 systèmes enzymatiques chez le mil.

En utilisant les techniques décrites ci-dessus, nous avons pu faire l'inventaire des locus codant pour ces 12 enzymes. Ainsi nous avons dénombré 48 locus dont l'expression est régulière et répétable dans nos conditions expérimentales. Seul l'isozyme POX-G ne peut pas être utilisé comme marqueur, en effet son expression varie suivant les conditions de croissance des plantes.

Les 48 locus étudiés mettent en jeu 109 allèles différents. 42 de ces locus sont polymorphes et présentent au moins 2 allèles. Signalons les locus pluri-alléliques les plus remarquables : un locus (EST E) possède 7 allèles, 2 locus (MDH B et PGI) ont 5 allèles.

Pour 27 d'entre eux, il s'agit de cas de dominance d'un allèle fonctionnel sur un allèle nul. Les 15 autres locus possèdent des allèles codominants capables de synthétiser au moins 2 types fonctionnels de protéines enzymatiques. 6 locus ne possèdent qu'une seule forme allélique (ils sont donc monomorphes) présente chez toutes les populations étudiées.

Le déterminisme génétique des locus est déduit de l'analyse des zymogrammes; pour certains d'entre eux il a été confirmé par l'étude de ségrégation $F_{2}$ (tabl. 3).

Comparons maintenant nos résultats à ceux obtenus par d'autres auteurs sur le mil :

La MDH a été étudiée par TOSTAIN \& RIANDEY (1985) qui ont trouvé 4 locus alors que nous en avons trouvé 5 dont un présent uniquement chez les feuilles vertes. La correspondance entre leur nomenclature et la nôtre est la suivante :

Notre locus - A ( 2 allèles) correspond au locus D (5 allèles).

Notre locus A (3 allèles) n'a pas été étudié par ces auteurs.

Notre locus B (5 allèles) correspond au locus A (5 allèles).

Notre locus C (2 allèles) correspond au locus B ( 3 allèles).

Notre locus D (3 allèles) correspond au locus C ( 3 allèles).

La différence importante qui sépare nos résultats de ceux de ces auteurs est le nombre d'allèles du locus - A : nous trouvons 2 allèles alors qu'ils en ont dénombré 5 .

Pour Catalase et ADH B on obtient les mêmes résultats que TOSTAIN \& RIANDEY (1984). Il n'en est pas de même pour le locus $\mathrm{A}$ de $\mathrm{ADH}$ où ces auteurs dénombrent 8 allèles en travaillant à $2 \mathrm{pH}$ différents (8 et 6), la mobilité électrophorétique de 5 d'entre eux étant sensible aux variations de $\mathrm{pH}$ alors que la mobilité des 3 derniers est identique à $\mathrm{pH} 6$ et 8 . Nos résultats ont été obtenus à $\mathrm{pH} 8,3$ et nous trouvons 3 niveaux de bandes alors que TOSTAIN \& RIANDEY en trouvent 6 , dont 3 très proches. Nos résultats sont en accord avec ceux d'autres auteurs ayant travaillé sur le mil (LEBLANC, 1978 ; BANUETT-BOURILLON \& HAGUE, 1979).

Les résultats que nous avons obtenus sont comparables à ceux obtenus chez d'autres céréales et notamment le maïs (SCANDALIOS et al., 1975 ; SCANDALIOS, 1979 ; GOODMAN et al., 1980).

Ce travail d'inventaire doit être complété en confirmant le déterminisme génétique de chaque système enzymatique (étude des ségrégations $\mathrm{F}_{2}$ ou $\mathrm{BC}$ ) et la détermination des groupes de linkage entre ces gènes, d'une part, et des marqueurs morphologiques, d'autre part.

L'étude des populations et de la domestication du mil grâce à ces données sur le polymorphisme enzymatique sera présentée dans les prochains articles. 


\section{RÉFÉRENCES BIBLIOGRAPHIQUES}

Banuett-Bourillon F., Hague D. R., 1979. Genetic analysis of alcohol dehydrogenase isozymes in pearl millet (Pennisetum typhoides). Biochem. Genet., 17, 537-552.

Bui Dang Ha D., Pernès J., 1982. Androgenesis in pearl millet. I. Analysis of plant obtained from microspore culture. Z. Pflanzenphysiol., 108, 317-327.

Cardy B. H., Stuber C. W., Goodman M. M., 1980. Techniques for starch gel electrophoresis of enzymes from maize (Zea mays L.). Inst. Stat., mimeogr. $\mathrm{n}^{\circ} 1317$. N. C. State Univ., Raleigh, N. C. Clément J., 1985. Les mils pénicillaires de l'Afrique de l'ouest. Prospection et collecte IBPGR-ORSTOM, $231 \mathrm{p}$.

Goodman M. M., Stuber C. W., Lee C. N., Johnson F. M., 1980. Genetic control of malate dehydrogenase isozymes in maize. Genetics, 94, 153-168.

IUPAC-IUB, 1973, Enzyme nomenclature, recommendations (1972) of the International Union of Pure and applied Chemistry and the international Union of Biochemistry. Elsevier Sci. Pub. Co., Amsterdam.

Leblanc J. M., 1978. Etude sur le système des Alcooldéshydrogénases du mil: Pennisetum typhoides (americanum). Thèse $3^{\mathrm{e}}$ cycle. Univ. Paris XI-Orsay, $61 \mathrm{p}$.

Leblanc J. M., Pernès J., 1983. Enzyme polymorphism of Pennisetum americanum in the Ivory Coast. Jap. J. Genet., 58, 121-131.

Lefranc-Riandey M. F., 1984. Variabilité allélique du système Alcool Déshydrogénase (ADH) chez le mil (Pennisetum americanum) ; mise en évidence de classes d'électromorphes par différentes conditions de migration. C. R. Acad. Sci., Paris, t. 299, Série III. $n^{\circ} 8,321-326$.

Ornstein L., 1964. Disc electrophoresis. Ann. N. Y. Acad. Sci., $121,321-347$.

Pernès J., 1983. Point de vue génétique sur la domestication des céréales. La Recherche, 146, 910-919.
Salanoubat M., 1980. Contribution à la connaissance du photopériodisme du mil (Pennisetum americanum) par l'étude de caractères floraux et de marqueurs enzymatiques. Mem. D.E.A. Dévelonnement et Amélioration des Végétaux, Univ. Paris X1-Orsay, 17

Sandmeier M., Beninga M., Pernès J., 1981. Analyse des relations entre formes spontanées et cultivées chez le mil à chandelles. III. Etude de l'hérédité des estérases et des péroxydases anodiques. Agronomie, 1, 487-494.

Scandalios J. C., 1969. Genetics control of multiple molecular forms of enzymes in plants. A review. Biochem. Genet., 3, 37-79.

Scandalios J. C., 1979. Control of gene expression and enzyme differentiation. In Scandalios J. G.: "Physiological Genetics", Acad. Press, N. Y., London, 280 p.

Scandalios J. C., Sorenson J. G., Ott L. A., 1975. Genetic control and intracellular localisation of glutamate oxaloacetate transaminase in maize. Biochem. Genet., 13, 759-763.

Stuber C. W., Goodman M. M., Johnson F. M., 1977. Genetic control and racial variation of glucosidase isozymes in maize (Zea mays L.). Biochem. Genet., 15, 383-394.

Tostain S., Riandey M. F., 1984. Polymorphisme et déterminisme génétique des enzymes de mil (Pennisetum glaucum L.). Etude des alcool déshydrogénases, catalases, endopeptidases et estérases. Agron. Trop., 39, 335-345.

Tostain S., Riandey M. F., 1985. Polymorphisme et déterminisme génétique des enzymes du mil pénicillaire (Pennisetum glaucum). Etude des malates déshydrogénases. Agronomie, 5, 227-238.

Trigui N., 1984. La variabilité génétique des mils (Pennisetum typhoides (Bur.) Stapf \& Hubb. de Tunisie : Etude biométrique et analyse du polymorphisme enzymatique. Thèse $3^{\mathrm{e}}$ cycle (Développement et Amélioration des Végétaux), Univ. Paris XI-Orsay, 125 p. 